\title{
On Requirements-driven Design of Distributed Smart Grid Automation Control
}

\author{
Chen-Wei Yang ${ }^{1}$, Valeriy Vyatkin ${ }^{1,2}$ \\ ${ }^{1}$ Dept. of Computer Science, Electrical and Space Engineering, Lulea University of Technology, Lulea, Sweden \\ ${ }^{2}$ Dept. Electrical Engineering and Automation, Aalto University, Helsinki, Finland \\ chen-wei.yang@ltu.se, vyatkin@ieee.org
}

\begin{abstract}
This paper proposes a novel method of modelling requirements for smart grid automation systems that fits and complements the leading standards used for software development in smart grid: IEC 61850 and IEC 61499. Ontological representation is used to formally model the systems requirements and the integration of the requirement modelling to be used in conjunction with IEC 61850 specifications and IEC 61499 implementation. The proposed model is illustrated on a case study presented by the CIGRE working group where the system requirement in natural language was firstly modelled in ontology, then implemented in IEC 61499. Lastly, the resulting IEC 61499 control system was tested and validated against the system requirement by signal testing of the end control system.
\end{abstract}

Keywords-IEC 61850, IEC 61499, Smart Grid, Ontology, Requirements Modelling

\section{INTRODUCTION}

Cyber physical systems (CPS) are a collection of physical and computing processes interacting via a communication network. Smart grids is an example of a complex CPS [1] with many distributed physical components interacting with distributed computing devices and processes. The interactions between the physical and the logical processes are enabled with the introduction of digital communication [2] and bi-directional energy flow [3] in the smart grid. The physical components of a smart grid are made up of sensors equipment which measures telemetry measurements such as voltages, currents and actuation equipment such as circuit breakers or switches for protection or control processes. The logical processes of a smart grid are made up of protection and control services running across the entire smart grid. Examples of protection services includes fault and safety management services and example of control processes includes energy and smart load management services. These logical processes are traditionally implemented on singular intelligent electronic devices (IED). However, with the greater integration of renewable energy resources and the greater interaction between the utilities and consumers, a centralized control architecture will no longer be sufficient. Therefore, in a smart grid architecture where the physical and the logical entities becomes more distributed and less centralized, there is a need to addresses the new design challenges of the emerging smart grid with the shift in infrastructure from centralized to distributed and this applies for both the physical and the logical component of the smart grid. Example of the design challenges includes the need of modelling the distributed physical architecture, modelling of the distributed logical process and interoperable communication network between the myriads of independent physical and logical processes.

On the automation side, IEC 61499 is an industrial standard which provides a reference architecture for designing and modelling of distributed control systems [4]. IEC 61499 is adept in modelling modular logical process for distributed control systems with basic modelling artefacts known as function blocks (FB). IEC 61499 introduces object orientation and top-down design approach to the modelling of automation control systems. IEC 61499 is widely adopted in other automation domains such as material handling, factory automation and building automation systems [5]. In addition, IEC 61499 also been used to smart grid applications where it is used to model the IEC 61850 specifications for the automation system $[6,7]$.

IEC 61850 is a substation automation standard with the aim to introduced communication interoperability to distributed substation systems. IEC 61850 introduces the concept of logical nodes (LN) which models physical (e.g. primary equipment) and functional (e.g. protection functions) specifications and each $\mathrm{LN}$ has a pre-define dataset that is necessary to model the said function or physical specification. Interoperability is achieved by standardizing the communication interface and the communication protocols within the substation. In addition to communication interoperability, IEC 61850 also introduces life cycle modeling of substations by means of the Substation Configuration Language (SCL) [8]. The SCL models cover the design life cycle from the specification stage to the testing stage as shown in Fig. 1. This introduces a pathway for utilizing model driven engineering (MDE) design techniques in the design of IEC 61850 based control systems. However, the SCL only introduces MDE at the specification level, whereas engineering lifecycle begins at the requirement level as illustrated in Fig. 1. Therefore, there is a need for the ability to formally model system requirements that can lead to the design flow specified in the SCL specifications.

System requirements are typically written in natural languages. However, natural language is not easily understandable or interpretably by machines and is also prone to ambiguities in the human interpretation, which could be catastrophic and costly if discovered deep in the design process. Formal modelling of system requirements can reveal these ambiguities early and create a format which is machine 


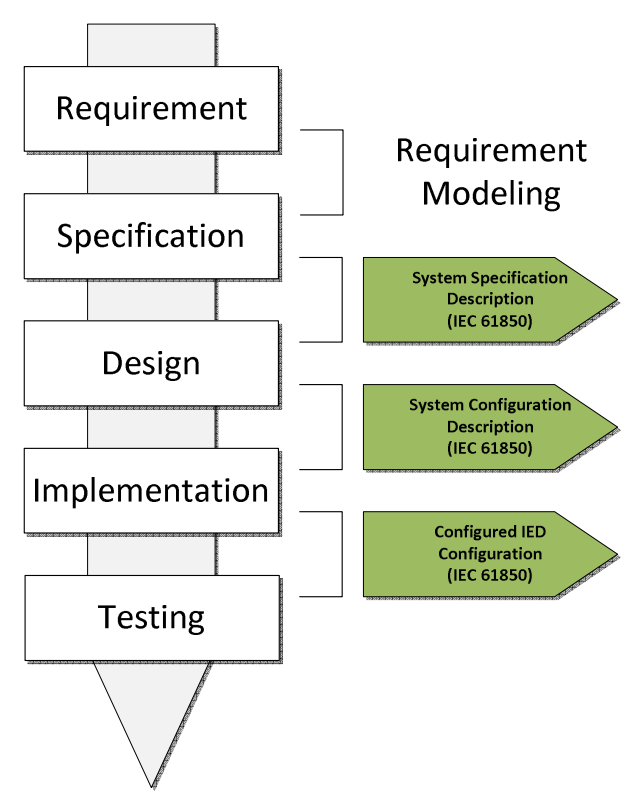

Fig. 1. Windfall engineering life-cycle for substation design

readable and therefore, can be integrated into the IEC 61850 design flow and used in conjunction with the SCL specifications.

The scope of the paper covers the formal modelling of substation system requirements using ontology models for the semantic web, the implementation of the formal requirement in IEC 61499 using IEC 61850 specifications and lastly, the validation of the IEC 61499 control system against the system requirement.

The paper is structured in the following way. Section II discusses the related works in requirement engineering in automation systems, Section III defines the ontology model for the modelling of the requirements. Section IV introduces the case study distributed system from CIGRE which is used to illustrate the proposed modelling process. This section covers the modeling of one of the system requirement in ontology, the implementation of the requirement in IEC 61499 based on IEC 61850 specifications. Lastly, the paper is concluded in section $\mathrm{V}$ which discusses the result of the paper and possible future works.

\section{RELATED WORKS}

In [10], the cost-efficient methods and processes for safety relevant (CESAR) requirements meta-modelling is adopted for a distributed protection system for the smart grid. The requirements are modelled using the boiler-plates methodology which provides semi-formal modelling of requirements. Boilplates represent a defined set of structures which can be used to describe requirements in a standardized method. However, it is not clear how the modelling of the requirements in the boilerplates leads to the executable specification, such as IEC 61499 since the use of the ontology model in this work is for the purposes of tracing the resulting IEC 61499 system and the requirement.
There are also works on semi-automated transformation methodologies which focus on transforming case studies or formal requirement models from the natural language representation. In [11], a methodology is presented in which natural language requirements are transformed to use-case diagrams in UML. The transformation identifies the use-case actors within the natural language description and creates context use-case diagram based on the contextual comparison model. In [12], a semi-automatic transformation methodology is proposed which aims to automatically transform natural language representation of requirements to boiler-plates which are used for requirement formalism since they support automated requirement analysis and is a semi-guided representation of the natural language requirement. The transformation is aided using domain ontology to correct typographic errors in natural language. The use of ontology is to correct typography errors in the transformation and acts as a reference dictionary whereas in this paper, the ontology is used to formally model the requirement itself.

In [13], a method to automatically generate test cases from system requirement for CPS system based on model driven engineering (MDE) technique is proposed. The proposed methodology uses various linked ontology models in the whole design chain in order to trace the development of the system at each step of the design process.

In summary, there are existing works on the application and modelling of system requirements in the research field. Within the smart grid domain, the formal modelling of system requirements is still lacking. This paper adopts the use of ontology for modelling system requirements for smart grid applications and focuses on integration of requirement engineering to IEC 61850.

\section{REQUIREMENT MODELING IN ONTOLOGY}

\section{A. Motivation}

The motivation behind this work arose from the CIGRE taskforce 5.02 meeting in June 2016 in Paris where the aim of the discussion was to examine the existing methods which are best suited for the modelling of functional requirements which can be an additive to the IEC 61850 design chain and the testing of the system requirement.

It is important at this stage to make a clear distinction of the differences between requirement and specification to illustrate why IEC 61850 is not considered a requirement modelling language. A requirement describes a behaviour or role of the system and hence, describes WHAT the system needs. An example of a system requirement is as follows:

Transformer should be tripped during overload currents superior to $120 \%$ on any of its coils

Which defines a certain behavior or role the system must perform with no inkling of how this role can be implemented. A specification defines HOW the requirement can be implemented. An example of a specification with respect to the requirement above is as follows: 
An overcurrent protection can be implemented on the primary and secondary side of the transformer to detect the overload

Which specifies the implementation of the overcurrent protection function on both the primary and the secondary side of the transformer to detect the overload. This specification can be modelled in IEC 61850 with the LNs Protection Instantaneous Overcurrent (PIOC) for overcurrent protection function. It's not possible to model requirements in IEC 61850 since the LN modelling artefacts falls under the category of specification definition rather than requirements since the LNs model a certain functionality or physical equipment.

In addition to the modelling of the system requirement, it is also paramount to be able to validate the correctness of the end system with respect to the initial design requirement and the most common software testing technique that is used for validating requirement in black box testing. IEC 61499 can model the specification, the implementation and black box testing of the implemented control system. There are existing research works which implements IEC 61850 specifications using IEC 61499 [6, 14, 15]. IEC 61499 is used as the modelling and implementation language for system requirement, IEC 61850 specification and validation in the research work in this paper.

\section{B. Modeling requirements in ontology}

Ontology is a modelling language for the semantic web which is adept in defining the knowledge of a domain with concepts and the relationships between the concepts. The advantages of modelling requirements using ontology models include:

- Defining the individual statement within a requirement and how these components are related to each other;

- Forcing the requirement engineers to explicitly state each statement in the requirement to avoid all ambiguities as illustrated in the case study in section IV;

- Can be queried with query languages such as semantic web rule language (SWRL) or SPARQL inferencing notation (SPIN) for resolving ambiguities and conflicts in natural language based requirements through ontology reasoning;

- Can be interpreted by machines with standard

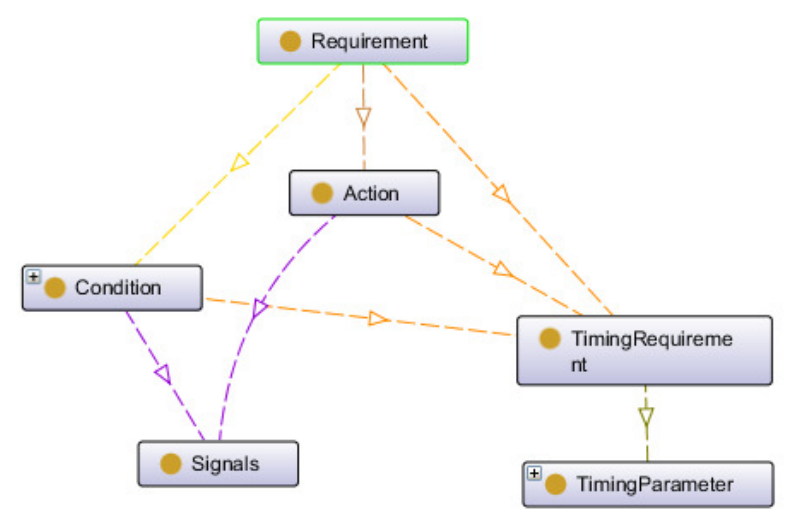

Fig. 2. Requirement domain and its concepts. representation such as resource description framework (RDF) and extensible markup language (XML).

In addition, ontology modelling are used extensively in IEC 61499 [16] based research work and IEC 61850 [17, 18] research work.

The ontology model of the Requirement domain and its concept are defined as:

\section{Requirement $\equiv$ Condition $\sqcup$ Action $\sqcup$ TimingRequirement}

Where Requirement is the main domain under the root node of the ontology. Condition, Action and TimingRequirement are all concepts under the Requirement domain linked by the object properties as shown in Fig. 2. The square boxes with a yellow circular symbol are the class concepts and the dashed arrows between the class concepts are the object properties when modelled in Protégé [19].

The Requirement domain contains one data property and three object properties. The naming convention of the properties are as follows: The object properties are defined as Has_<objectPropertyName $>$ and the data objects are defined as has_<dataPropertyName $>$.

The data property has_name contains the name of the Requirement and the cardinality is exactly 1 :

$$
\text { (= } 1 \text { has_name) }
$$

The object properties Has_Action, Has_Condition and Has_TimingRequirement indicates the number of Actions, Conditions and Timing Requirement of the Requirement and the cardinality for the object properties are all at minimum 0 :

$$
\begin{gathered}
(\geq 0 \text { Has_Condition } \sqcap \geq 0 \text { Has_Action } \sqcap \geq \\
\text { Has_TimingRequirement) }
\end{gathered}
$$

The first concept under the domain Requirement is TimingRequirement, which defines the timing requirement. There can be timing requirements at different levels of the requirement, be it at the level of conditions, actions or the requirement itself. The concept TimingRequirement has one object property and two data properties.

The data properties are has_name and has_description, which describes the name of the timing requirement and the description of the timing requirement respectively. The cardinality of both data properties is exactly 1 :

$$
\text { (= } 1 \text { has_name } \sqcap=1 \text { has_description) }
$$

The object property of the concept TimingRequirement is Has_TimingParameter linking to the concept

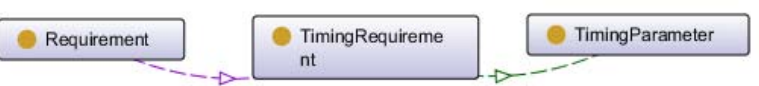

Fig. 3. Timing Requirement and its associated concepts.

TimingParameter as shown in Fig. 3. The cardinality for Has_TimingParameter is exactly 1 : 


\section{( = 1 Has_TimingParameter)}

The concept TimingParameter has two data properties which are has_at_least and has_within providing the timing constraint of the timing requirement. The has_at_least data property specifies the minimum quantity of time in which the condition, action or the requirement must elapse. The has_within data property specifies the maximum quantity of time in which the condition, action or the requirement must elapse within. Both data properties have a cardinality of 1 .

$$
\text { (= } 1 \text { has_at_least } \sqcup=1 \text { has_within) }
$$

The second concept under the domain Requirement is condition, which outlines the conditions of the requirement. The condition concept has two data properties, has_description and has_name which describes the condition and the name of the condition respectively. The cardinality of the data properties is exactly 1 :

$$
\text { (= } 1 \text { has_name } \sqcap=1 \text { has_description) }
$$

The object properties of the concept condition are Has_TimingRequirement and Has_Signals which associates condition with the concepts TimingRequirement and Signals as shown in Fig. 4. The cardinality of both these object properties are at minimum 0 :

\section{( $\geq 0$ Has_TimingRequirement $\geq$ Has_Signals)}

The concept Signals defines the signals for the conditions, and actions. The concept Signal has two data properties,

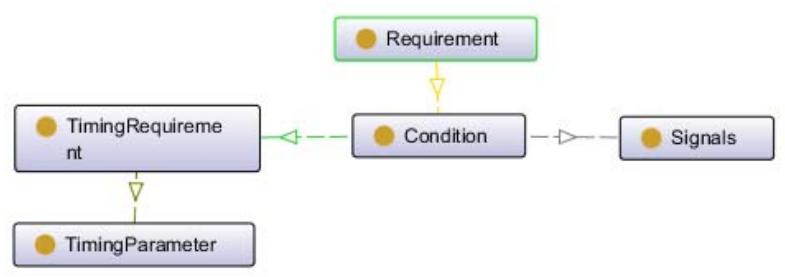

Fig. 4. Condition and its associated concepts.

has_input_signal and has_output_signal which defines the input signal and the output signals respectively. The cardinality of these two data properties are at minimum 0 :

$$
\text { ( } \geq 0 \text { has_input_signal } \sqcap \geq 0 \text { has_output_signal) }
$$

The last concept under the Requirement domain is Action. The Action concept has the same data properties and object properties to that of the condition concept. The data properties are has_description and has_name with cardinality of exactly 1:

$$
\text { (= } 1 \text { has_name } \sqcap=1 \text { has_description) }
$$

The two object properties are Has_TimingRequirement and Has_Signals which associates the Action concept with the

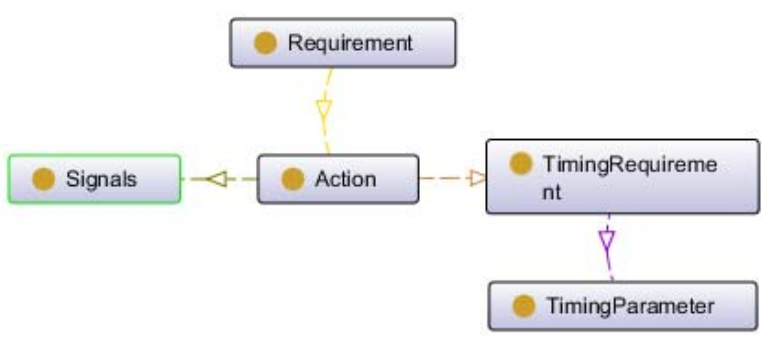

Fig. 5. Action and its associated concepts.

concepts TimingRequirement and Signal respectively as shown in Fig. 5.

\section{CASE STUdY}

\section{A. A simple distribution system}

The case study that will be a simple distribution system with three requirements to be modelled as shown in Fig. 6 was presented during the CIGRE taskforce meeting. There is a transformer TRl with two current transformers, $C T 1$ and $C T 2$ measuring the primary and the secondary current of the transformer. On the primary side, there are two switches $S W 11$ and SW12 along with a circuit breaker BRK1. On the secondary side, there are also two switches $S W 21$ and $S W 22$ along with a circuit breaker $B R K 2$.

\section{B. System requirements in natural language}

The most readily understandable form of requirements is natural language. The three requirements which will be used for this case study as described in natural languages are as follows:

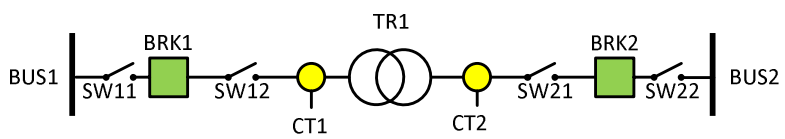

Fig. 6. Simple distribution system.

(1) Transformer TR1 should be tripped during overload currents superior to $120 \%$ on any of its coils that stays for at least $50 \mathrm{~ms}$ in no more than 100ms.

(2) Opening or closing of switches SW11 and SW12 shall be blocked whenever breaker BRK1 stays closed.

(3) Any unauthorized access to the automation or protection system shall be detected, alarmed, recorded and signaled to the operator in no more than 1 min after its detection.

Although requirements in natural language can be readily interpreted by domain practitioners, ambiguity can still occur and an example of this is requirement (1) pertaining to the timing constraint "no more than $100 \mathrm{~ms}$ ". There are two ways in which this can be interpreted. The first interpretation is that the tripping of Transformer TRl should be completed within $100 \mathrm{~ms}$ and this does not include the detection time. This 
means that the timing constraint is an action level timing constraint according to the ontology definition. The second interpretation is that the process of overload detection and tripping must all occur within $100 \mathrm{~ms}$ and this means the timing constraint is a requirement level timing constraint. Modelling the requirements in ontology can clean up these ambiguities and correctly define the requirements.

\section{Modeling in ontology}

Each primary equipment in the case study system can be modelled as signals either as the type BOOLEAN or INTEGER. For all switches SW11, SW12, SW21 and SW22, the SWITCH.pos and SWITCH.act of type BOOLEAN are defined and the designation are as follows:

1. The SWITCH.pos signal are input signals which indicate the positional value of the switches. A value of TRUE means the switch is in the Closed position and a value of FALSE indicates the switch is in the Opened position.

2. The SWITCH.act signal are output signals which issues the actuation command to the switches. A value of TRUE means to close the switch and a value of FALSE means to open the switch.

For the current transformers CT1 and CT2, an input signal of type INTEGER is used to represent the primary and the secondary current readings of the TR1 transformer. For the breakers BRK1 and BRK2, the same definition is applied akin to that of the switches where BREAKER.pos is an input signal indicating the position of the breaker, BREAKER.act is an output signal issuing the actuation command and both are of type BOOLEAN. A summary of all the signal definitions is found in Table 1.

Table 1. Summarization of signals.

\begin{tabular}{|c|c|c|}
\hline Name & Direction & Description \\
\hline SW11.pos: BOOL & Input & $\begin{array}{l}\text { Position signal of Switch SW11 } \\
\text { where TRUE = Closed state and } \\
\text { FALSE = Opened state }\end{array}$ \\
\hline SW12.pos: BOOL & Input & Same as above \\
\hline SW21.pos: BOOL & Input & Same as above \\
\hline SW22.pos: BOOL & Input & Same as above \\
\hline SW11.act: BOOL & Output & $\begin{array}{l}\text { Actuation signal of switch SW11 } \\
\text { where TRUE = to CLOSE and FALSE } \\
=\text { to OPEN }\end{array}$ \\
\hline SW12.act: BOOL & Output & Same as above \\
\hline SW21.act: BOOL & Output & Same as above \\
\hline SW22.act: BOOL & Output & Same as above \\
\hline CT1: INT & Input & Current reading of CT1 \\
\hline CT2: INT & Input & Current reading of $\mathrm{CT} 2$ \\
\hline BRK1.pos: BOOL & Input & $\begin{array}{l}\text { Position signal of Breaker BRK1 } \\
\text { where TRUE = Closed state and } \\
\text { FALSE = Opened state }\end{array}$ \\
\hline BRK2.pos: BOOL & Input & Same as above \\
\hline BRK1.act: BOOL & Output & $\begin{array}{l}\text { Actuation signal of Breaker BRK1 } \\
\text { where TRUE = to CLOSE and FALSE } \\
=\text { to OPEN }\end{array}$ \\
\hline BRK2.act: BOOL & Output & Same as above \\
\hline
\end{tabular}

Due to page constraints, only the ontology model for the first system requirement is discussed in this paper. Examining requirement 1 closely, the requirement can be broken down into a series of conditions and actions.

Condition 1: Overload currents superior to $120 \%$ on any of its coils

Condition 2: Condition 1 stays for at least 50ms

Action 1: Transformer TR1 should trip when Condition 1 and Condition 2 are satisfied in no more than 100ms

In total, there are two conditions and one action. The two conditions are satisfied sequentially before the action can be performed. Requirement 1 when modelled in the ontology model is shown in Fig. 7. The rectangular boxes with a purple filled diamond symbol are the class individuals. The solid arrow lines show the relationships between the class concept and its individuals and the dashed arrow lines shows the object properties between the class individuals. Requirement_l has two Has_Condition object properties associated to the Condition_1 and Condition_2 individual, Has_Action object property associated to action Action_l and a data property has_name with the value of "Requirement_1".

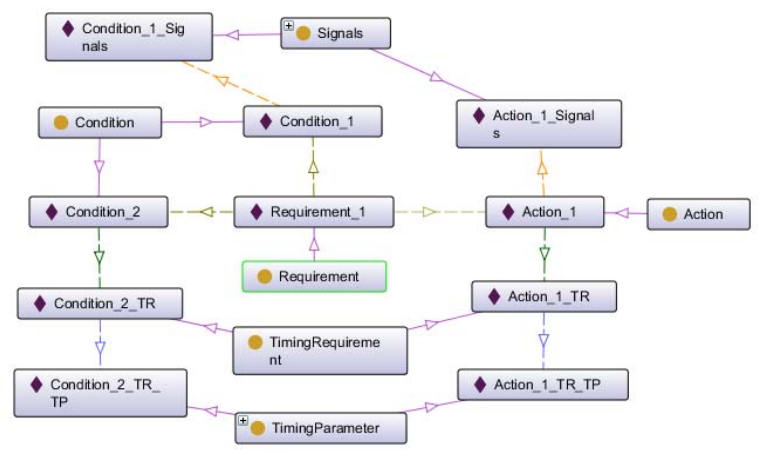

Fig. 7. Requirement 1 modeled in ontology.

The first condition, Condition_l when modelled in ontology is shown in Fig. 0. The Condition_l ontology has the following assertion properties and property values:

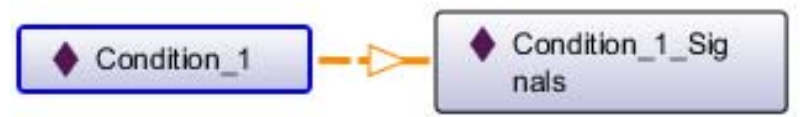

Fig. 8. Condition_l and its associated individuals.

Object Properties:

1. Has_Signal Condition_1_Signals

\section{Data Property:}

1. has_name "C1"

2. has_description "Overload current superior to $120 \%$ on any of its coils"

The object property Has_Signal means there are a set of signals for Condition_1. The has_description retains the description of the condition in natural language.

The Signal individual Condition_1_Signals has three data properties: 


\section{Data Property:}

1. has_input_signal "CT1"

2. has_input_signal "CT2"

3. has_input_signal "overload_Threshold"

Signals CT1 and CT2 measure the current on the primary and the secondary side of transformer TR1. The signal overload_threshold sets the required overload percentage.

The ontology model of the secondly condition, Condition_2 is shown in Fig. 9. The property assertions and its values are as follows:

\section{Object Properties:}

1. Has_TimingRequirement Condition_2_TR

\section{Data Property:}

1. has_name "C2"

2. has_description "Condition 1 stays for at least 50ms"

The object property Has_TimingRequirement indicates that Condition_2 has a timing requirement Condition_2_TR. The associated property assertions for the timing requirement are as follows:

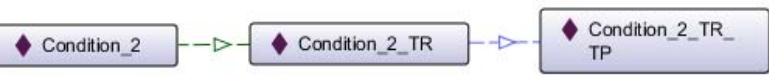

Fig. 9. Condition_2 and its associated individuals.

\section{Object Properties:}

1. Has_TimingParameter Condition_2_TR_TP

Timing requirement $\mathrm{C} 2$ has a timing parameter Condition_2_TR_TP. The timing parameter has the following data properties:

\section{Data Property:}

\section{1. has_at_least "50ms"}

The data property adds the timing constraint to condition_1 which means condition_2 is satisfied when condition_l is true for more than $50 \mathrm{~ms}$.

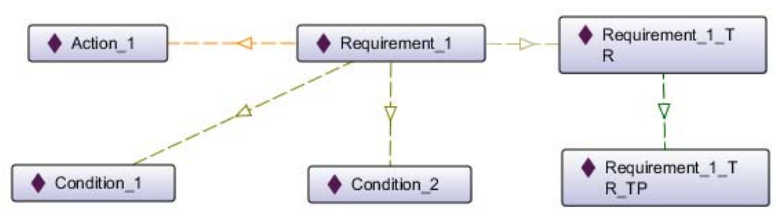

Fig. 10. Alternate interpretation of Requirement 1 where the timing requirement is on the requirement level.

The ontology model of the action Action_l is shown below in Fig. 11. The property assertions are as follows:

\section{Object Properties:}

1. Has_TimingRequirement Action_1_TR

2. Has_Signal Action_1_Signal

\section{Data Property:}

$$
\text { 1. has_name "A1" }
$$

2. has_description "Transformer TR1 should trip when Condition 1 and condition 2 are satisfied in no more than $100 \mathrm{~ms}$ "

The action Action_l has two object properties. The first object property Has_Signal indicates Action_l has a set of signals Action_1_Signal. The property assertions of Action_1_Signal are as follows:

\section{Data Property:}

1. has_output_signal "BRK1.act"

2. has_output_signal "BRK2.act"

The two output signals for Action_1_Signal are BRK1.act and BRK2.act which trips either BRK1 or BRK2 depending on whether the overload is on the primary side or the secondary side of the transformer TR1.

The second object property Has_TimingRequirment means Action_l has one timing requirement and the property assertions for Action_1_TR are:

\section{Object Properties:}

1. Has_TimingParameter Action_1_TR_TP

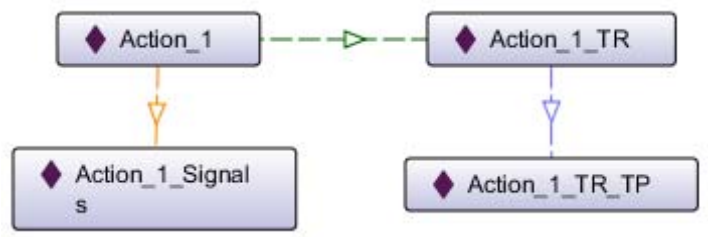

Fig. 11. Action_1 and its associated individuals.

The property assertions of the timing parameters Action_1_TR_TP are as follows:

\section{Data Property:}

\section{1. has_within "100ms"}

The data property has_within shows the timing in which the action must be performed within.

Note that as discussed earlier in section IV, sub section $\mathrm{C}$, there are two possible interpretations of the second timing requirement in the first requirement. The first interpretation is that the tripping must be completed within $100 \mathrm{~ms}$ after the overload fault is detected and this is the ontology in Fig. 7 depicts this interpretation where this timing requirement is on the level of Action_1. The second interpretation is that the detection and the tripping must occur within 100ms and the ontology model which depicts this interpretation is in Fig. 10 where the timing requirement is on the level of Requirement_1. This illustrates one of the advantages of modelling requirements in ontology where it is necessary to explicitly state each component of the requirement to avoid ambiguities introduced in the natural language representation.

\section{Requirement Implementation}

Requirement modeling is the first step in the engineering process. However, it is also important to verify whether the end system satisfies the system requirements. IEC 61499 is the reference standard for distributed automation control system and is capable of modeling high level design abstraction of 


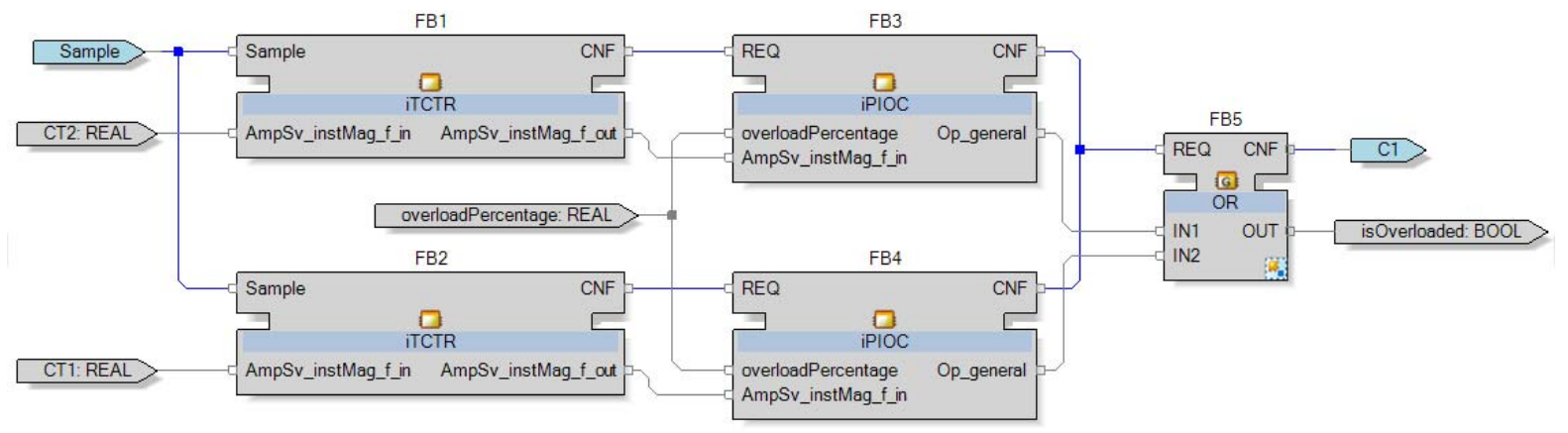

Fig. 12. IEC 61850 implementation of Condition 1.

requirements and specifications. The main design artefacts in IEC 61499 are FB blocks as shown in Fig. 13. There are two types of FB artifacts. A basic FB and a composite FB. All FB artefacts consists of a set of input and output interfaces with event and data connections. Within a basic FB artefact, there can either be an Execution Control Chart (ECC), which implements the functional control of the FB whereas in a composite FB can encapsulate a network of interconnected FBs.

Each condition and action within a system requirement can be modelled in a composite FB artefact as shown in Fig. 13. Condition 1 as defined earlier in the paper is:

Condition 1: Overload currents superior to $120 \%$ on any of its coils.

In the FB model of Condition 1, there are three data inputs and one data output. The two data inputs are CT1 and CT2. CT1 and CT2 are the current measurement readings of the primary and the secondary side of the transformer coil as defined in Table 1. The third input is the parametrization input overloadPercentage, which sets the overload percentage. The output data is isOverloaded, which indicates whether an overload is detected on the coils.

The implementation of condition $\mathrm{C} 1$ is shown in Fig. 12. It is based on the Logical Node (LN) models from the IEC 61850 standard where LNs are used to model functional specifications. The iTCTR FB is based on the current transformer LN Current Transformer (TCTR) and it is used to collect the current reading at each sampling step. In Fig. 12, two iTCTR FB instances are used since it is necessary to collect the current measurement from the primary and the secondary side of the transformer. The iPIOC FB is modelled from the overcurrent LN PIOC and it checks whether the current sample of the measurement reading exceeds the pre-set overload percentage. If an overload is detected, the output $O p \_$general will be set to TRUE and FALSE if no overload current is detected. The last FB is an OR logical operator which outputs TRUE if an overload is detected on either side of the transformer.

The complete implementation of requirement 1 in $\mathrm{FB}$ is shown in Fig. 13. There are three FBs representing Condition 1 (C1), Condition 2 (C2) and Action 1 (A1). The C2 FB implements condition 2 which checks for whether the $\mathrm{C} 1$ is satisfied for $50 \mathrm{~ms}$ as defined in requirement 1 . If $\mathrm{C} 2$ is satisfied, the trip output will be set to TRUE. Otherwise, it will be set to FALSE. The A1 FB performs Action 1, which will actuate the Breakers if $\mathrm{C} 1$ and $\mathrm{C} 2$ are satisfied.

\section{E. Testing and validation of requirement in end system}

It is essential to validate the correctness of the end control system with respect to the system requirement. There are two testing techniques that are commonly used in software engineering. Black box testing, also called behavioral and functional testing, is used to validate the end control system and to examine whether the high-level design corresponds to the initial requirement specifications while white-box testing is used to verify the system by testing the internal implementation of the system specifications. The principle of

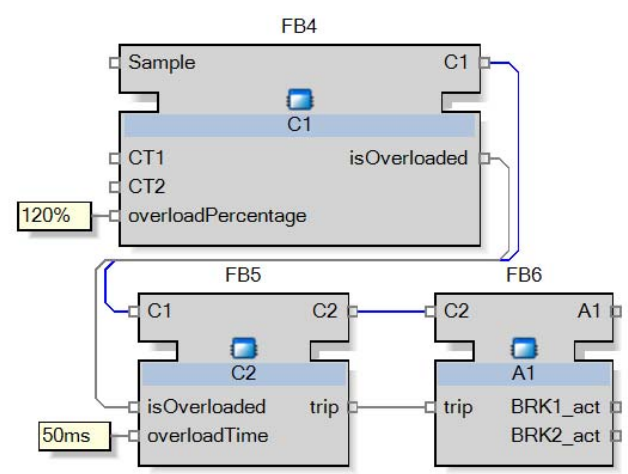

Fig. 13. FB Implementation of Requirement 1.

black box testing is to disregard the internal implementation of the functionality and solely focus on the outputs that are generated based on the selected inputs into the system to test whether the intended behavioral requirement is implemented in the end system. In IEC 61499 , it is possible to perform black box requirement testing by creating scripting FBs which perform various testing scenario to test the end control system.

For example, to test validate requirement 1 , the following testing scenario can be written:

1. A fault signal is inserted into the primary side of TR1,

2. The fault is inserted for $70 \mathrm{~ms}$.

The FB implementation of this test scenario is shown on the left of Fig. 14. The R1_Test test case FB has two events, InsertPrimaryFault and InsertSecondaryFault which 
generates fault current to the primary side and the secondary side of the TRl transformer respectively. The parametrization value of faultTime sets the time in which the fault is active.

The R1_Composite FB on the right side in Fig. 14 is the composite FB representation of Requirement 1 from Fig. 13. $C T 1$ and $C T 2$ are the current reading of primary and secondary coil of TR1. The overloadPercentage input sets the overload percentage. The overloadTime input parametrizes the needed overload time before a tripping signal can be emitted. In the test scenario shown in Fig. 14, a fault is generated at the primary side of transformer $T R 1$ for $90 \mathrm{~ms}$ with the InsertPrimaryFault event input. This will insert a fault current into the R1_Composite FB for 90ms. On the output of the R1_Composite FB, The BRK1_act output indicates a tripping signal has been emitted to the breaker on the primary coil and the trip signal was emitted $3 \mathrm{~ms}$ after the confirmation of the overload fault.

This is one example of how black box testing of requirements can be performed in IEC 61499. There are other testing techniques such as the close loop co-simulation testing in [20] where a dedicated power system simulator was used to test the control system. This co-simulation framework was extended in [21] where MATLAB scripting was used to allow automated script testing of the control system under the same co-simulation environment.

\section{CONCLUSION}

The aim of this paper is to propose a methodology to formally model substation automation system (SAS) requirements from natural language, which can be supplemented to the SAS engineering process introduced in the IEC 61850 standard. An ontology model is proposed to formally model SAS system requirements with the advantage

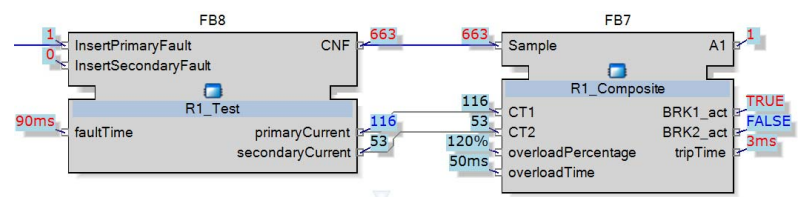

Fig. 14. Black box testing of requirement 1. Test case FB (Left) Requirement 1 FB (Right).

of minimizing ambiguities in the natural language representation of system requirements and in addition, is machine interoperable and can be integrated in MDE driven design approaches such as [18]. The proposed approach is demonstrated in a distribution system with system requirements defined in natural language. The natural language representation is firstly modelled in ontology to clear the ambiguity in the requirement by breaking the requirement down into conditions and actions. Then, an IEC 61499 representation of the ontology requirement is developed which can be used for functional implementation of requirements based on IEC 61850 LNs and in addition, performing black box testing of end IEC 61499 system with respect to the initial system requirements.
The future work is to investigate how an IEC 61499 application could be automatically generated based solely from the requirement ontology. This could reduce engineering time in the design process since the overall structure of the control code can be automatically created. This could also be extended further to allow the automatic generation of the implementation of the specification using IEC 61850 with respects to the requirement FBs.

\section{REFERENCES}

[1] H. Gharavi, H. Chen, and C. Wietfeld, "Guest Editorial Special Section on Cyber-Physical Systems and Security for Smart Grid," Smart Grid, IEEE Transactions on, vol. 6, no. 5, pp. 2405-2408, 2015.

[2] X. Fang, S. Misra, G. Xue, and D. Yang, "Smart Grid - The New and Improved Power Grid: A Survey," Communications Surveys \& Tutorials, IEEE, vol. 14, no. 4, pp. 944-980, 2012.

[3] A. Ipakchi and F. Albuyeh, "Grid of the future," IEEE Power and Energy Magazine, vol. 7, no. 2, pp. 52-62, 2009.

[4] Function Blocks-Part 1 Architecture, International Electrotechnical Commission.

[5] V. Vyatkin, "IEC 61499 as Enabler of Distributed and Intelligent Automation: State-of-the-Art Review," Industrial Informatics, IEEE Transactions on, vol. 7, no. 4, pp. 768-781, 2011.

[6] N. Higgins, V. Vyatkin, N.-K. C. Nair, and K. Schwarz, "Distributed Power System Automation With IEC 61850, IEC 61499, and Intelligent Control," Systems, Man, and Cybernetics, Part C: Applications and Reviews, IEEE Transactions on, vol. 41, no. 1, pp. 81-92, 2011.

[7] C.-W. Yang, G. Zhabelova, V. Vyatkin, N.-K. Nair, and A. Apostolov, "Smart Grid automation: Distributed protection application with IEC61850/IEC61499," in Industrial Informatics (INDIN), 2012 10th IEEE International Conference on, 2012, pp. 1067-1072

[8] IEC 61850 - Communication networks and systems for power utility automation, IEC Standard, Part 6: Configuration language for communication in electrical substations related to IEDs - Ed 2, 2009.

[9] S. Rohjans, C. Dänekas, and M. Uslar, "Requirements for Smart Grid ICTarchitectures," in 2012 3rd IEEE PES Innovative Smart Grid Technologies Europe (ISGT Europe), 2012, pp. 1-8.

[10] R. Sinha, S. Patil, C. Pang, V. Vyatkin, and B. Dowdeswell, "Requirements engineering of industrial automation systems: Adapting the CESAR requirements meta model for safety-critical smart grid software," in IECON 2015 - 41 st Annual Conference of the IEEE Industrial Electronics Society, 2015, pp. 002172-002177.

[11] S. M. Seresht and O. Ormandjieva, "Automated assistance for use cases elicitation from user requirements text," Proceedings of the 11th Workshop on Requirements Engineering, pp. 128-139

[12] S. Farfeleder, T. Moser, A. Krall, T. Stålhane, H. Zojer, and C. Panis, "DODT: Increasing requirements formalism using domain ontologies for improved embedded systems development," in 14th IEEE International Symposium on Design and Diagnostics of Electronic Circuits and Systems, 2011, pp. 271-274.

[13] R. Sinha, C. Pang, G. S. Martinez, and V. Vyatkin, "Automatic test case generation from requirements for industrial cyber-physical systems," AtAutomatisierungstechnik, vol. 64, pp. 216-230, 2016.

[14] G. Zhabelova and V. Vyatkin, "Multiagent Smart Grid Automation Architecture Based on IEC 61850/61499 Intelligent Logical Nodes," IEEE Transactions on Industrial Electronics, vol. 59, no. 5, pp. 2351-2362, 2012.

[15] T. Strasser, M. Stifter, F. Andren, D. Burnier de Castro, and W. Hribernik, "Applying Open Standards and Open Source Software for Smart Grid Applications: Simulation of Distributed Intelligent Control of Power Systems," in IEEE Power \& Energy Society General Meeting 2011, 2011.

[16] W. Dai, V. Dubinin, and V. Vyatkin, "Automatically Generated Layered Ontological Models for Semantic Analysis of Component-Based Control Systems," Industrial Informatics, IEEE Transactions on, vol. 9, no. 4, pp. 2124-2136, 2013.

[17] R. Santodomingo, S. Rohjans, M. Uslar, J. A. Rodríguez-Mondèjar, and M. A. Sanz-Bobi, "Facilitating the Automatic Mapping of IEC 61850 Signals and CIM Measurements," IEEE Transactions on Power Systems, vol. 28, no. 4, pp. 4348-4355, 2013.

[18] C.-W. Yang, V. Dubinin, and V. Vyatkin, "Ontology Driven Approach to Generate Distributed Automation Control from Substation Automation Design," IEEE Transactions on Industrial Informatics, vol. PP, no. 99, pp. 1-1, 2016.

[19] Protégé Available: http://protege.stanford.edu.

[20] C. H. Yang, G. Zhabelova, C.-W. Yang, and V. Vyatkin, "Co-Simulation Environment for Distributed Controls of SmartGrid," Industrial Informatics, IEEE Transactions on, vol. PP, no. 99, pp. 1-1, 2013.

[21] N. Kashyap, C.-W. Yang, S. Sierla, and P. G. Flikkema, "Automated Fault Location and Isolation in Distribution Grids With Distributed Control and Unreliable Communication," IEEE Transactions on Industrial Electronics, vol. 62, no. 4, pp. 26122619 , 